\title{
Application of MLP and RBF Methods in Prediction of Travelling within the city
}

\author{
Mehdi Nosrati ${ }^{1, *}$, Mojtaba Hoseini ${ }^{2}$, Alireza ShIRMARz ${ }^{2}$, Abbas Mirzaei Somarin ${ }^{3}$, \\ Nayereh HOSEININIA ${ }^{1}$, Morteza BARARI ${ }^{2}$ \\ ${ }^{1}$ Department of Computer Engineering, Payame Noor University(PNU), P.O.Box, \\ 19395-3697 Tehran, Iran \\ ${ }^{2}$ Faculty of Information, Communications and Security Technology, Malek Ashtar \\ University of Technology, Tehran, Iran \\ ${ }^{3}$ Department of Computer Engineering, Islamic Azad University, Ardebil Branch, \\ Ardebil, Iran
}

\begin{abstract}
Forecasting of Travelling within the city demand is necessary for the correct operations of subway stations. This includes the provision of station security, management and better service to passengers will be. In this paper we are compared multilayer perceptron(MLP) and Radial Basis Function (RBF) models together for prediction of travelling within the city. The models are trained and assessed on dataset of Aliabad subway station in Tehran.
\end{abstract}

Keywords: Urban Travels, Artificial Neural Network, multilayer perceptron, Radial Basis Function.

\section{Introduction}

Forecasting of urban travels demand is necessary for the correct operations of subway stations. This forecasting is usually used for planning, such as determining the Personnel subway station, management, better service to passengers and station security. Urban travel statistics can be considered as time series. Many techniques exist for the approximation of the underlying process of a time series: linear methods such as ARX, ARMA, etc.[1,2], and nonlinear ones such as artificial neural networks $[1,3]$. The common difficulty to all the methods is the determination of sufficient and necessary information for an accurate prediction. There is a great attention to new approaches for the enhancement of forecasting accuracy because of economical and industrial aspects. ARIMA models [4,5] are one of the traditional approaches for forecasting issues. ARIMA models and the other classic approaches such as Kalman Filters suffer from nonlinear behavior of dynamical systems[8]. Artificial Neural networks (NNs) [3],[6-9] have succeeded in several power system problems, such as planning, control, analysis, protection, design, load forecasting, security analysis, and fault diagnosis[10]. Artificial neural networks are being applied to forecasting problems since their distributed structure of weights and neurons permits to approach complex relationships between variables without specifying them explicitly in advance. Since that time series of urban travel system has many ebbs and flows, therefore, focus of this paper is new methods of non-linear that the results of this study show that the accuracy of these methods. In this paper the Multi Layerd Perceptron(MLP) and Radial Basis Function(RBF) models are compared together. The input data consists of sequences of Aliabad subway station of Tehran dataset. The file 
contain daily urban travels. The rest of the paper is outlined as follows. Section 2 and 3 describes Multilayer Perceptron Network and Radial Basis Function respectively. Finally, Section 4, presents the simulation experimental results followed by Section 5 that concludes the paper.

\section{Multilayer Perceptron Network}

A neural network is massively parallel-distributed Processor made up of simple processing units called neurons, which have a natural propensity for storing experimental knowledge[11]. Using a feedforward artificial neural network to enable forecasting of urban travels, the choice was between the two most common types; multi-layer perceptrons (MLPs) and radial basis function (RBF) networks. The fundamental difference is the way in which hidden units combine values coming from preceding layers in the network, MLP's use inner products, while RBF's use Euclidean distance. A multi-Layer perceptrons network was created, It is one of the most important types of neural network models. It consists of a set of sensory nodes, the input layer, one or more hidden layers of computing nodes, and an output layer of computing nodes (Fig.1). They are commonly termed "Multilayer Feed Forward Networks. The input signal propagates through the network in a forward direction, hence the term feed-forward. They are trained using an algorithm known as the error back-propagation algorithm. This process is made up of two passes through the network layers, one forward and one backward. In the forward pass, the input is applied to the sensory nodes, and its effect propagates through the network layers, the response then appears at the output nodes. This output is compared against a desired value, to produce an error signal which then propagates backward through the network. In the forward pass, the synaptic weights of the network are fixed, but during the backward pass they are adjusted so that the network output moves closer towards the desired response (Fig.1).

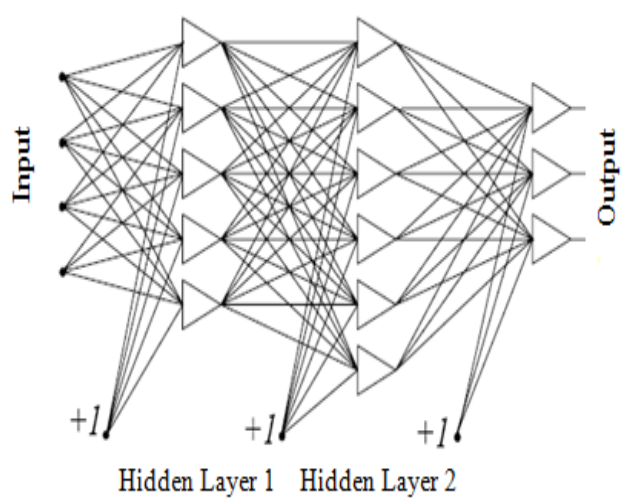

Fig. 1: The structure of Feedforward neural network.

\section{Radial Basis Function Network}

The RBF networks, just like MLP networks, can therefore be used classification and/or function approximation problems. RBFNs exhibit a good approximation and learning ability and are easier to train and generally converge very fast. The RBFN is a 3-layered feedforward network (Fig. 2) comprising of input, hidden/memory, and output neurons respectively. It uses a linear transfer function for the output units and Gaussian function (radial basis function) for the hidden units [12]. 
The RBFs, which have a similar architecture to that of MLPs, however, achieve this goal using a different strategy(Fig.2):

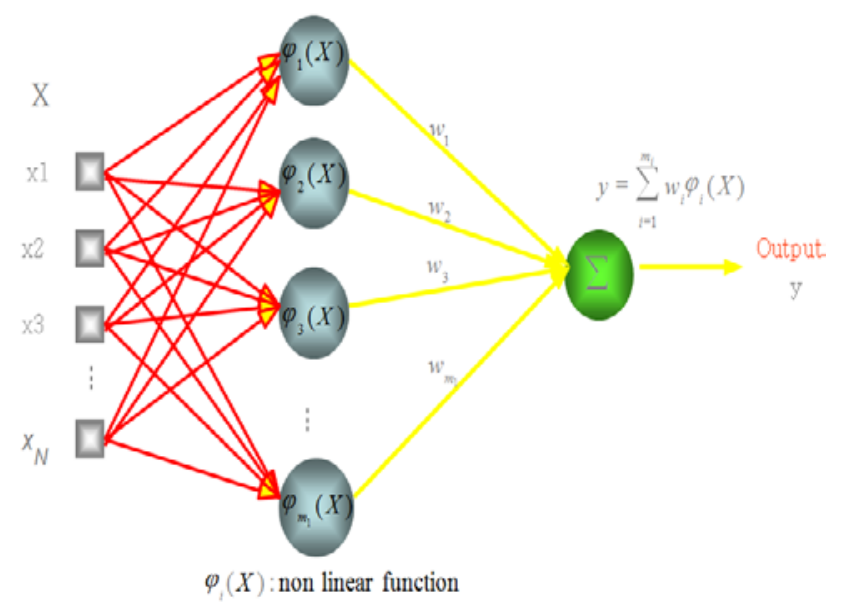

Fig.2: The structure of Radial Basis Function networks.

A hidden layer of radial kernels: The hidden layer performs a non-linear transformation of input space. The resulting hidden space is typically of higher dimensionality than the input space. An output layer of linear neurons: The output layer performs linear regression to predict the desired targets. Dimension of hidden layer is much larger than that of input layer Cover's theorem on the separability of patterns.

\section{Experiments}

\subsection{Dataset}

One time series is used as an example. The dataset is called Urban travels statistics, and it represents period of the daily travel of Aliabad Station during around 1360 days. The first 1300 values are used for training and testing, and the remaining data for prediction. The learning part of the dataset is shown in Fig. 3.

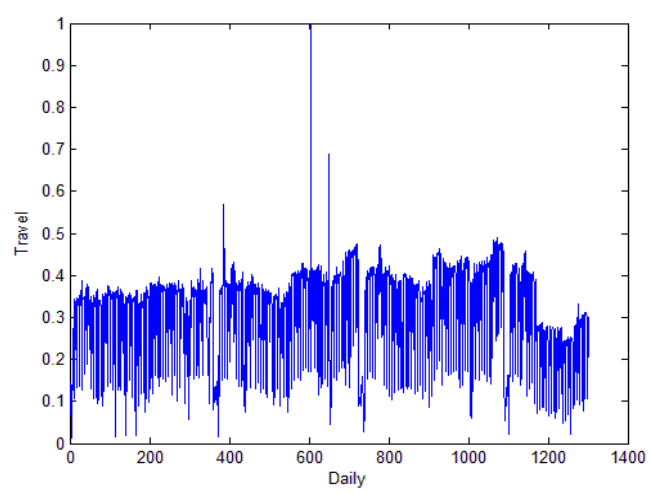

Fig.3 :.Learning set of the Urban Travels Statistics dataset.

\subsection{Results}

The general problem of time series forecasting can be rephrased as the problem of finding a model able to forecast the future evolution of a time series given its past 
evolution. The main reason motivating such approach is reliability of the predicted values. In this section the above models are trained and assessed on Urban Travels dataset. Fig. 4 depict the result achieved by the MLP, RBF models on daily forecasting. As can be seen the forecast Urban Travel curves produced by MLP model follow the actual ones and the results of MLP are better than RBF. Table 1 shows the forecasting errors - mean, maximum, and minimum absolute error, mean square error (MSE)- of these two models for daily urban travels. It can be inferred from fig. 4 and table 1 that the MLP model performance for this dataset is better than RBF model. They show that the model could not even learn the shape of the actual urban travels curves. That illustrates how difficult is the problem of predicting accurately peak of urban travel curves in daily horizons. According to the figures 4 and table 1 we can say that increasing in prediction horizon cause increasing prediction error.

Table 1: Forecasting errors - mean, maximum, and minimum absolute error, mean square error (MSE)- on daily urban travels.

\begin{tabular}{|l|l|l|l|l|}
\hline Error & Mean & Max & Min & MSE \\
\hline RBF & 0.0535 & 0.1764 & 0.0015 & 0.0085 \\
\hline MLP & 0.0532 & 0.1875 & 0.00035 & 0.0037 \\
\hline
\end{tabular}

\section{Conclusion}

In this paper we compared MLP with RBF for urban travels forecasting as a demanding practical problem. There models were trained and assessed on Urban Travels of Aliabad station dataset. The experiments showed that the MLP model performance for this dataset is better than RBF model.

\section{References}

1. Antti Sorjamaa, Jin Hao, Nima Reyhani, Yongnan Ji, Amaury Lendasse,” Methodology for long-term prediction of time series”, 2007 Elsevier.

2. L. Ljung, System Identification Theory for User, Prentice-Hall, Englewood Cliffs, NJ, 1987

3. A.S. Weigend, N.A. Gershenfeld, Times Series Prediction: Forecasting the future and Understanding the Past, Addison-Wesley, Reading, MA, 1994.

4. L. Ljung, System Identification Theory for User, Prentice- Hall, Englewood CliPs, NJ, 1987.Haykin, S.: Neural Networks: A Comprehensive Foundation, MacMillan, New York, 1994.

5. G.E.P. Box, G. Jenkins, Time Series Analysis: Forecasting and Control,Cambridge University Press, Cambridge, 1976.

6. M. Cottrell, B.Y. Girard, M. Mangeas, C. Muller, Neural modeling for time series: a statistical stepwise method for weight elimination, IEEE Trans. Neural Networks 6 (6) pp. 1355-1364, 1995

7. M. Cottrell, B. Girard, P. Rousset, Forecasting of curves using a Kohonen classification, J. Forecasting 17,pp. 429-439, 1998. 
8. A. Lendasse, E. de Bodt, V. Wertz, M. Verleysen, Nonlinear Financial time series forecasting - application to the Bel20 stock market index, European J. Econom. Social Systems 14 (1) , pp. 81-91, 2000.

9. J. Sjoberg, Q. Zhang, L. Ljung, A. Benveniste, B. Delyon, P.Y. Glorennec, H. Hjalmarsson, A. Juditsky, Non linear black box modeling in system identification: an unified overview, Automatica 33, pp. 1691-1724, 1997.

10. Ot’avio A.S. Carpinteiro, Rafael C. Leme , Antonio C. Zambroni de Souza ,Carlos A.M. Pinheiro , Edmilson M. Moreira ,"Long-term load forecasting via a hierarchical neural model with time integrators”, Elsevier B.V., 2006

11. Ummuhan Basaran Filik1, Mehmet Kurban,“A New Approach for the Short-Term Load Forecasting with Autoregressive and Artificial Neural Network Models", International Journal of Computational Intelligence Research. ISSN 0973-1873 Vol.3, No.1 (2007), pp. 66-71

12. Khan M.R. and Ondrusek C. (2000), Application of the radial basis function networks to the problem of short-term load forecasting", Journal "Elektryka”, No. 87, 11/12, PL ISSN 0373-8647, Technical University of Gdansk, Poland, pp. 3-14. 